\title{
Article \\ A WRKY Transcription Factor, EjWRKY17, from Eriobotrya japonica Enhances Drought Tolerance in Transgenic Arabidopsis
}

\author{
Dan Wang ${ }^{1,2}$, Qiyang Chen ${ }^{1}$, Weiwei Chen ${ }^{1,2}$, Xinya Liu ${ }^{1,2}$, Yan Xia ${ }^{1,2}$, Qigao Guo ${ }^{1,2}$, Danlong Jing ${ }^{1,2, *(\mathbb{D})}$ \\ and Guolu Liang ${ }^{1,2, *}$
}

1 Key Laboratory of Horticulture Science for Southern Mountains Regions of Ministry of Education, College of Horticulture and Landscape Architecture, Southwest University, Beibei, Chongqing 400715, China; wander007@email.swu.edu.cn (D.W.); cqy0909@email.swu.edu.cn (Q.C.); weiwei_chen15134@zzu.edu.cn (W.C.); barophiles@email.swu.edu.cn (X.L.); yansummer@swu.edu.cn (Y.X.); qgguo75@swu.edu.cn (Q.G.)

2 Academy of Agricultural Sciences of Southwest University, State Cultivation Base of Crop Stress Biology for Southern Mountainous Land of Southwest University, Beibei, Chongqing 400715, China

* Correspondence: jingdanlong@swu.edu.cn (D.J.); lianggl@swu.edu.cn (G.L.); Tel.: +86-023-68250383 (D.J.); +86-023-68250383 (G.L.)

Citation: Wang, D.; Chen, Q.; Chen, W.; Liu, X.; Xia, Y.; Guo, Q.; Jing, D.; Liang, G. A WRKY Transcription Factor, EjWRKY17, from Eriobotrya japonica Enhances Drought Tolerance in Transgenic Arabidopsis. Int. J. Mol. Sci. 2021, 22, 5593. https://doi.org/ $10.3390 /$ ijms 22115593

Academic Editor: Lam-Son Phan Tran

Received: 15 April 2021

Accepted: 18 May 2021

Published: 25 May 2021

Publisher's Note: MDPI stays neutral with regard to jurisdictional claims in published maps and institutional affiliations.

Copyright: (c) 2021 by the authors. Licensee MDPI, Basel, Switzerland. This article is an open access article distributed under the terms and conditions of the Creative Commons Attribution (CC BY) license (https:// creativecommons.org/licenses/by/ $4.0 /)$.

\begin{abstract}
The WRKY gene family, which is one of the largest transcription factor (TF) families, plays an important role in numerous aspects of plant growth and development, especially in various stress responses. However, the functional roles of the WRKY gene family in loquat are relatively unknown. In this study, a novel WRKY gene, EjWRKY17, was characterized from Eriobotrya japonica, which was significantly upregulated in leaves by melatonin treatment during drought stress. The EjWRKY17 protein, belonging to group II of the WRKY family, was localized in the nucleus. The results indicated that overexpression of EjWRKY17 increased cotyledon greening and root elongation in transgenic Arabidopsis lines under abscisic acid (ABA) treatment. Meanwhile, overexpression of EjWRKY17 led to enhanced drought tolerance in transgenic lines, which was supported by the lower water loss, limited electrolyte leakage, and lower levels of reactive oxygen species (ROS) and malondialdehyde (MDA). Further investigations showed that overexpression of EjWRKY17 promoted ABA-mediated stomatal closure and remarkably up-regulated ABA biosynthesis and stress-related gene expression in transgenic lines under drought stress. Overall, our findings reveal that EjWRKY17 possibly acts as a positive regulator in $\mathrm{ABA}-$ regulated drought tolerance.
\end{abstract}

Keywords: Eriobotrya japonica; WRKY17; overexpression; drought stress; ABA

\section{Introduction}

Plants often face multiple environmental constraints in terms of drought stress, temperature extremes, and salinity [1]. Water deficit is the main environmental factor severely constraining plant development and production. To adapt to these abiotic stresses, plants have evolved a series of intricate strategies at molecular, cellular, biochemical, and physiological levels [2,3], including stomatal movement, signal perception and transduction, the expression of stress-induced genes and activation of physiological and metabolic responses [4]. When plants are exposed to stress conditions, the transcription factors (TFs) act as central regulators by binding to specific cis-acting elements in the promoter regions to activate downstream gene expression, signal transduction, and adaptation networks [5]. To date, a wide range of TFs families in plants, including WRKY, NAC, MYB, and bHLH, have been functionally identified and elucidated to participate in abiotic stress in plants [6-9].

As one of the largest transcription factor families, WRKY TFs are crucial regulatory proteins that respond to biotic and abiotic stresses and modulate physiological processes and development [10]. The WRKY family, originally isolated from sweet potato, contain 
either 1 or 2 WRKY domains, which consist of a conserved WRKYGQK sequence motif and a zinc finger structure $\left(\mathrm{C}_{2} \mathrm{H}_{2}\right.$ or $\left.\mathrm{C}_{2} \mathrm{HC}\right)$ [11]. The WRKY gene family is classified into groups I, II, and III and different subsets based on the number of WRKY domains and the variation of zinc finger motif $[12,13]$. Proteins having two WRKY domains belong to group I, while groups II and III have a single WRKY domain, the latter two groups are distinguished by $\mathrm{C}_{2} \mathrm{H}_{2}$ and $\mathrm{C}_{2} \mathrm{HC}$, respectively [13]. The modulation of the transcription of downstream target genes can be regulated by WRKY TFs via specifically binding to the $\mathrm{W}$-box elements [(T)TGAC(C/T)] cis-elements [12]. Growing evidence has proved that WRKY proteins are involved in plant responses to a broad spectrum of abiotic stresses, such as drought, cold, and salt $[6,14,15]$. For instance, AtWRKY21, AtWRKY46, AtWRKY54, and AtWRKY70 in Arabidopsis have been reported to regulate osmotic stress [16-18]. TaWRKY2 transgenic wheat enhanced tolerance to drought stress, reduced the water loss rate of detached leaves, and increased grain yield [19]. In addition, overexpression of OsWRKY11 and OsWRKY30 effectively enhanced drought tolerance in transgenic rice seedlings [20,21]. AtWRKY57 increased resistance to drought in Arabidopsis through regulation of water loss rate and abscisic acid (ABA) content [22]. Overexpression of MfWRKY17 in Arabidopsis increased water retention, maintained chlorophyll content, and regulated the transcription levels of ABA biosynthesis and the stress-related genes, thus improving the resistance to drought stress [23].

Loquat (Eriobotrya japonica Lindl.), belonging to the Rosacea family, is one of the semitropical fruit trees widely distributed in Southeastern China [24,25]. However, the growth, development, and yield of loquat are often affected by multiple abiotic stresses, especially drought and cold stresses [26]. Our previous research showed that the WRKYs family was involved in the drought stress in loquat seedlings [27]. However, the function and molecular mechanism of WRKY TF family members in loquat remains elusive. Here, a novel WRKY gene was cloned from Eriobotrya japonica and designated as EjWRKY17. The ectopic expression of EjWRKY17 positively regulates drought tolerance in transgenic Arabidopsis through ABA-mediated stomatal closure, inhibition of reactive oxygen species (ROS) accumulation, and up-regulation of the expression of stress-related genes. These results deepen the understanding of the WRKY TFs' functions in loquat in response to drought stress.

\section{Results}

\subsection{Identification of EjWRKY17 from Eriobotrya japonica}

A WRKY transcription factor gene named EjWRKY17 was isolated from Eriobotrya japonica based on the homology cloning and RACE techniques. The open reading frame of EjWRKY17 is $837 \mathrm{bp}$, encoding 278 amino acids (Genebank accession number: MW528209). The calculated molecular weight (MW) was $30.5 \mathrm{kD}$ and the isoelectric point (PI) of the deduced protein was 9.82. As shown in Figure 1A and Figure S1, multiple alignments of amino acid sequences showed that EjWRKY17 protein contained a conserved WRKY domain, consisting of a WRKYGQK motif and a $\mathrm{C}_{2} \mathrm{H}_{2}$ zinc-finger-like motif, which was similar to several WRKY17s with known functions in different plant species. In addition, a total of seven conserved functional domains were identified in the EjWRKY17 protein. Motif 1 was defined as the WRKY domain, and a nuclear localization signal (NLS) (KRRK) was found in Motif 2. Additionally, a conserved C-motif and HARF motif were found in Motifs 4 and 5, respectively. Therefore, EjWRKY17 can be classified into group II of the WRKY family. In Rosacea plants, the protein sequence of EjWRKY17 was most homologous to MdWRKY11 and MdWRKY17 of Malus domestica (Figure 1B). EjWRKY17 was phylogenetically closer with VvWRKY17 of Vitis vinifera than with AtWRKY17 of Arabidopsis, MfWRKY17 of Myrothamnus Flabellifolia. 
A

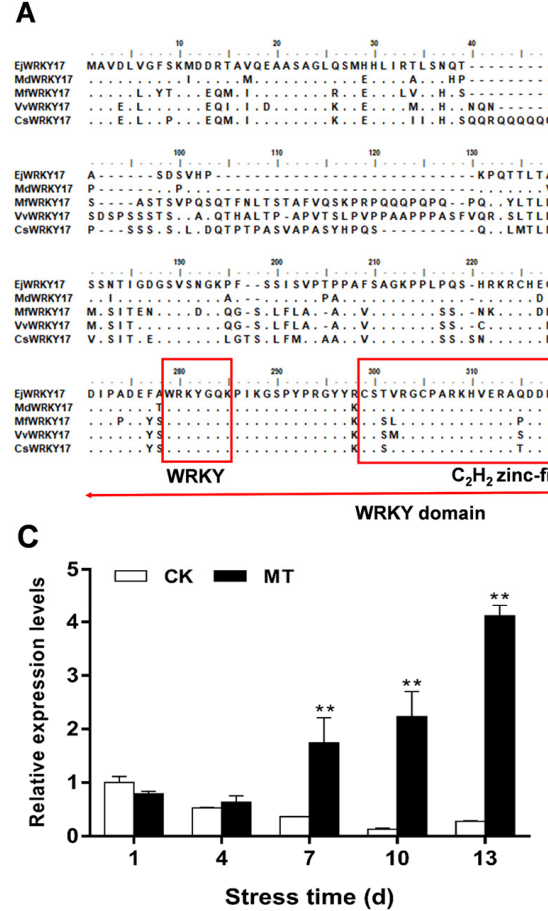

B

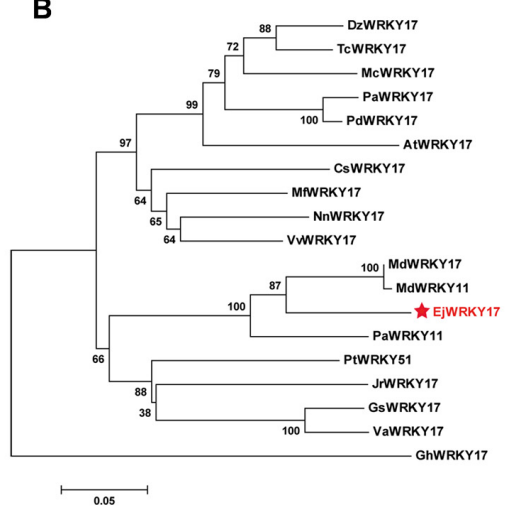

D
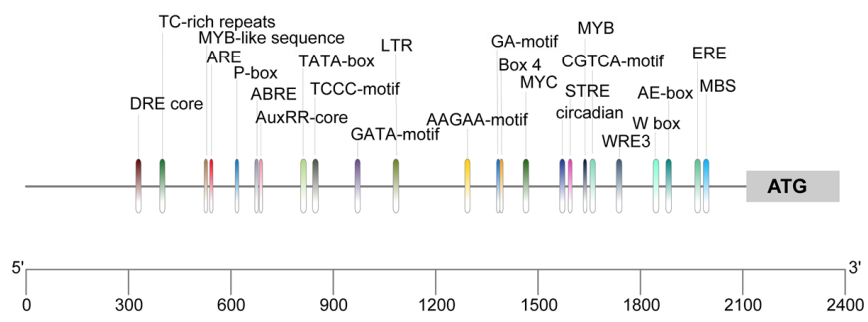

Figure 1. Characterization of EjWRKY17. (A) Multiple alignment of amino acid sequences of EjWRKY17 with other WRKY proteins. (B) Phylogenetic analysis among EjWRKY17 and WRKY proteins from other plant species. (C) Expression levels of EjWRKY17 during drought stress. (D) Cis-acting elements in the promoter region of EjWRKY17 gene. The conserved domain or motif was marked by the red line; NLS, nuclear localization signal. Asterisks denote significant differences (as compared with the control group): ** $p<0.01$.

Based on our previous study, exogenous melatonin enhanced drought tolerance in loquat seedlings and significantly induced high expression of WRKY17 under drought stress at the transcriptome level. To verify the expression pattern of EjWRKY17 in response to water deficit, loquat seedlings pretreated with or without melatonin were exposed to drought for 13 days (d) and RT-qPCR assays were performed (Figure 1C). Under drought stress, EjWRKY17 expression in control leaves (CK) significantly decreased from $1 \mathrm{~d}$ to $10 \mathrm{~d}$ and then increased at $13 \mathrm{~d}$. In contrast, the expression of EjWRKY17 was induced from $7 \mathrm{~d}$ to $13 \mathrm{~d}$ in response to exogenous melatonin treatment and the expression levels in melatonintreated (MT) leaves were significantly higher than that of the control leaves from $7 \mathrm{~d}$ to $13 \mathrm{~d}$. These results suggested that EjWRKY17 gene may be positively involved in the drought resistance of loquat. In addition, we investigated the promoter region of EjWRKY17 gene for the presence of specific cis-acting elements (Figure 1D and Table S1). The results showed that the EjWRKY17 promoter contains one MYB binding site (MBS) involved in drought inducibility, one dehydration responsive element (DRE core), one defense and stressresponsive element (TC-rich repeats), and one element related to ABA responsiveness (ABRE), indicating that the EjWRKY17 might be involved in plants' response to ABAmediated drought stress.

\subsection{Subcellular Localization of EjWRKY17 Protein}

In order to examine the subcellular location of the EjWRKY17, we constructed 35S::EjWRKY17GFP fusion controlled by CaMV 35S promoter. The recombined fusion vector and the control vector (35S::GFP) were transferred into the young leaves of tobacco (Nicotiana benthamiana) by Agrobacterium-mediated transformation. Then the transgenic leaves were stained with 4,6-diamidino-2phenylindole (DAPI). As shown in Figure 2, the green fluorescence of the control was localized both in the nucleus and cytoplasm, while the signal of the 35S::EjWRKY17-GFP was only targeted to the nucleus. These findings revealed that the EjWRKY17 is a nuclear-localized protein. 

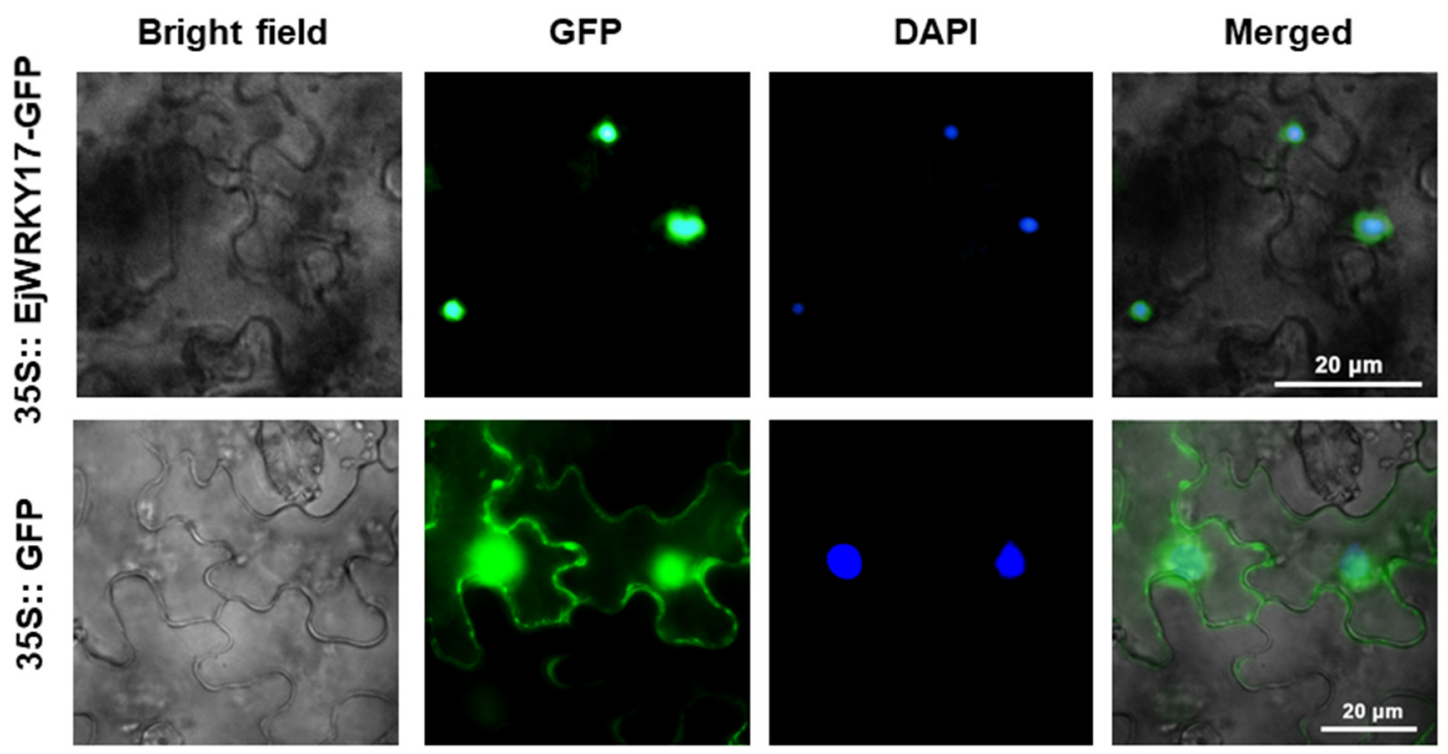

Figure 2. The subcellular localization of EjWRKY17 in N. benthamiana epidermal cells. GFP: Green fluorescence protein. DAPI: Nucleic marker. Merged: Merged image of bright field, GFP, and DAPI.

\subsection{Overexpression of EjWRKY17 Increased ABA Tolerance in Arabidopsis}

$\mathrm{ABA}$, as a major chemical signal, plays a crucial role in regulating abiotic stress responses [28]. To observe the response of EjWRKY17 transgenic plants to ABA treatment, Arabidopsis seeds of the T3 generation of WT and EjWRKY17 overexpression lines (L3 and L4) were surface-sterilized and germinated on half-strength MS medium supplemented with $\operatorname{ABA}(0,0.5$, and $1 \mu \mathrm{M})$. As the concentration increased, ABA treatment inhibited the germination rate of EjWRKY17 transgenic and WT plants. However, the germination rate was almost identical between the WT and transgenic lines with or without ABA treatment (Figure 3A,B). After six days of growth, there was no significant difference in germination rate between EjWRKY17 transgenic line and WT plants in the presence of 0.5 or $1.0 \mu \mathrm{M}$ $\mathrm{ABA}$. Interestingly, the rates of green cotyledon in both EjWRKY17 transgenic lines were significantly higher than that in the WT at both ABA concentrations (Figure 3C).

In addition, the sensitivity to ABA of EjWRKY17 overexpression lines was further determined at the post-germination stage. Under normal growth conditions, no phenotypic differences were observed between the WT and transgenic plants. The primary root lengths of Arabidopsis were inhibited by ABA stress with the increase of the treatment concentration (Figure 4). In the presence of $1 \mu \mathrm{M} A B A$, the root length of EjWRKY17 transgenic lines was $3.95 \mathrm{~cm}$, about $0.52 \mathrm{~cm}$ longer than that of WT plants. Under $5 \mu \mathrm{M}$ ABA treatment, a more pronounced inhibition of root length was observed in the WT plants compared to the EjWRKY17 overexpression lines. These results suggested that overexpression of EjWRKY17 enhanced tolerance to ABA at both germination and post-germination stages in Arabidopsis.

\subsection{Overexpression of EjWRKY17 Enhanced Drought Tolerance in Arabidopsis}

With the increase of the mannitol concentration, the root growth in WT plants was more severely inhibited than in EjWRKY17 transgenic plants (Figure 5). When exposed to $150 \mathrm{mM}$ mannitol stress, EjWRKY17 transgenic lines had notably longer root lengths than the WT line. In the presence of $300 \mathrm{mM}$ mannitol, the root lengths of L3 and L4 lines were constrained to 3.05 and $2.76 \mathrm{~cm}$, significantly higher than that of the WT plants. Taken together, these results indicated that overexpression of EjWRKY17 in Arabidopsis could enhance tolerance to mannitol-induced drought. 
$\mathbf{A}$

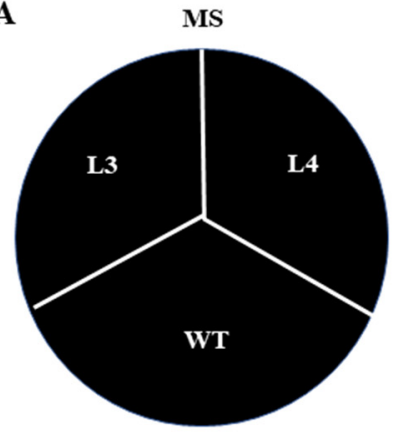

$0.5 \mu \mathrm{M} A B A$

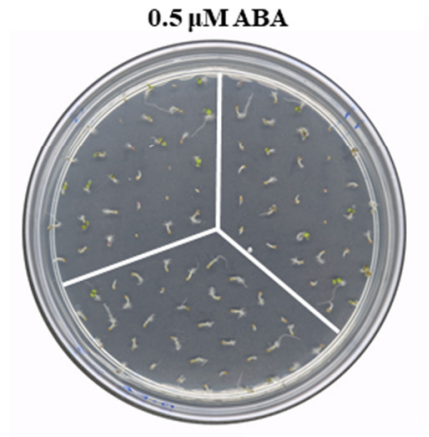

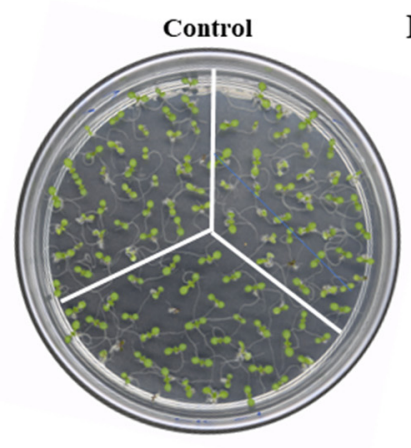

$1 \mu \mathrm{MABA}$

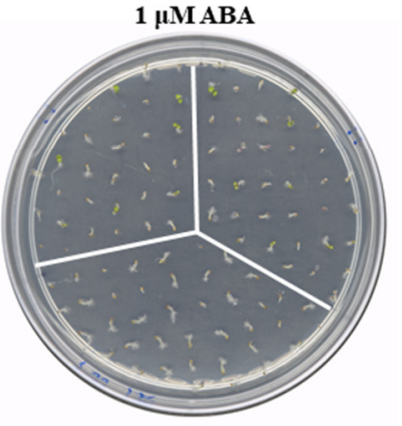

B

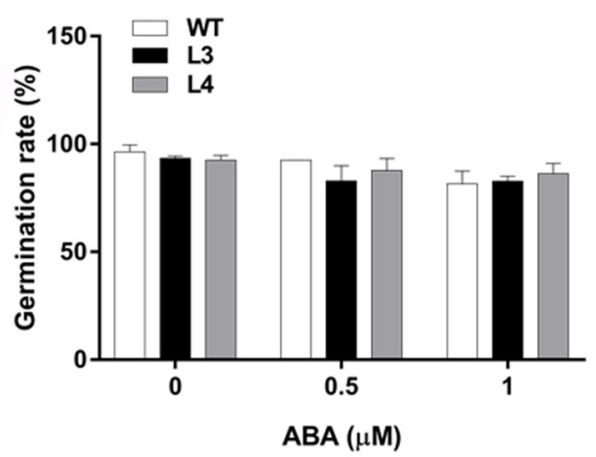

C

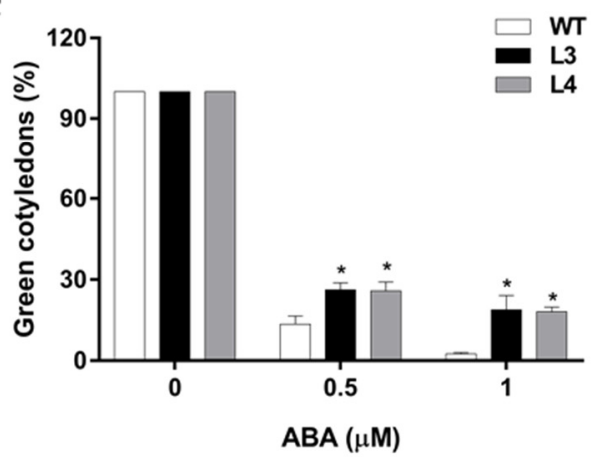

Figure 3. Germination and green cotyledon analysis of Arabidopsis under ABA treatment. (A,B) Germination rates. (C) Cotyledon greening rates. Error bars represent $\pm \mathrm{SD}(n=3)$. Asterisks denote significant differences (as compared with the control group): ${ }^{*} p<0.05$.
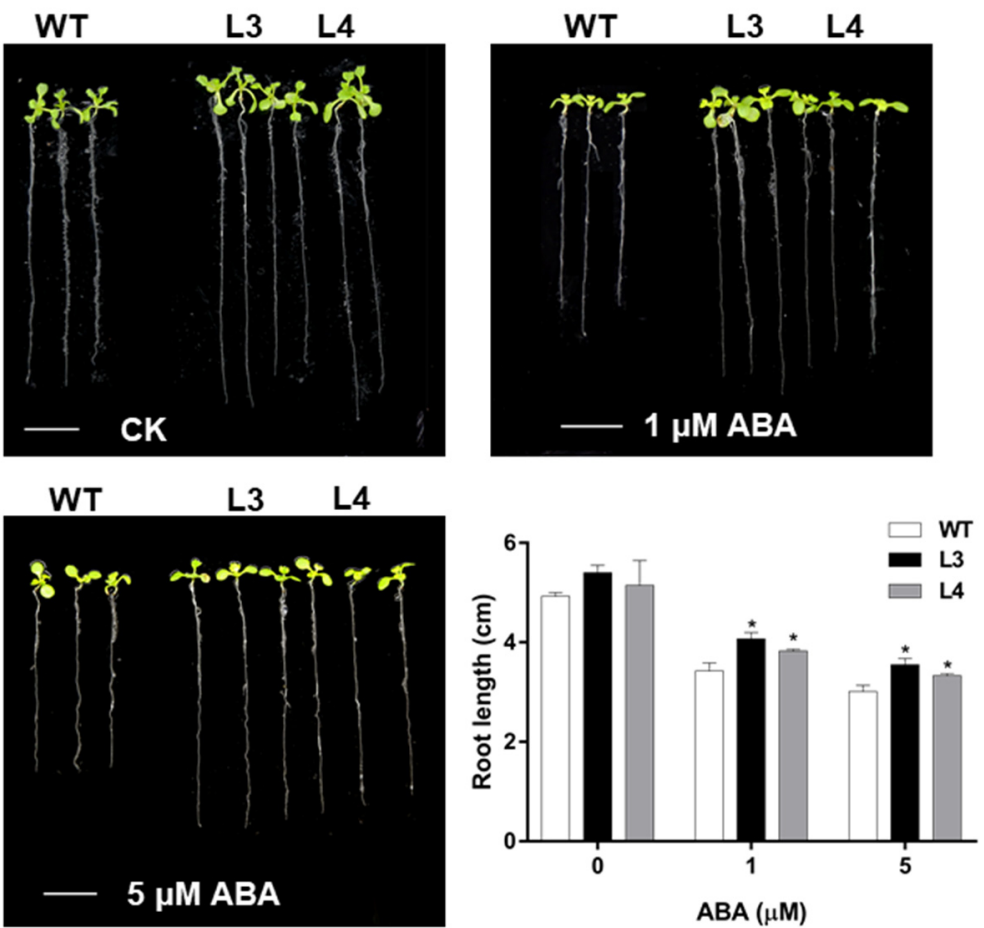

Figure 4. Root elongation analysis of Arabidopsis under ABA treatment. Error bars represent $\pm \mathrm{SD}$ $(n=3)$. Asterisks denote significant differences (as compared with the control group): ${ }^{*}<0.05$. 

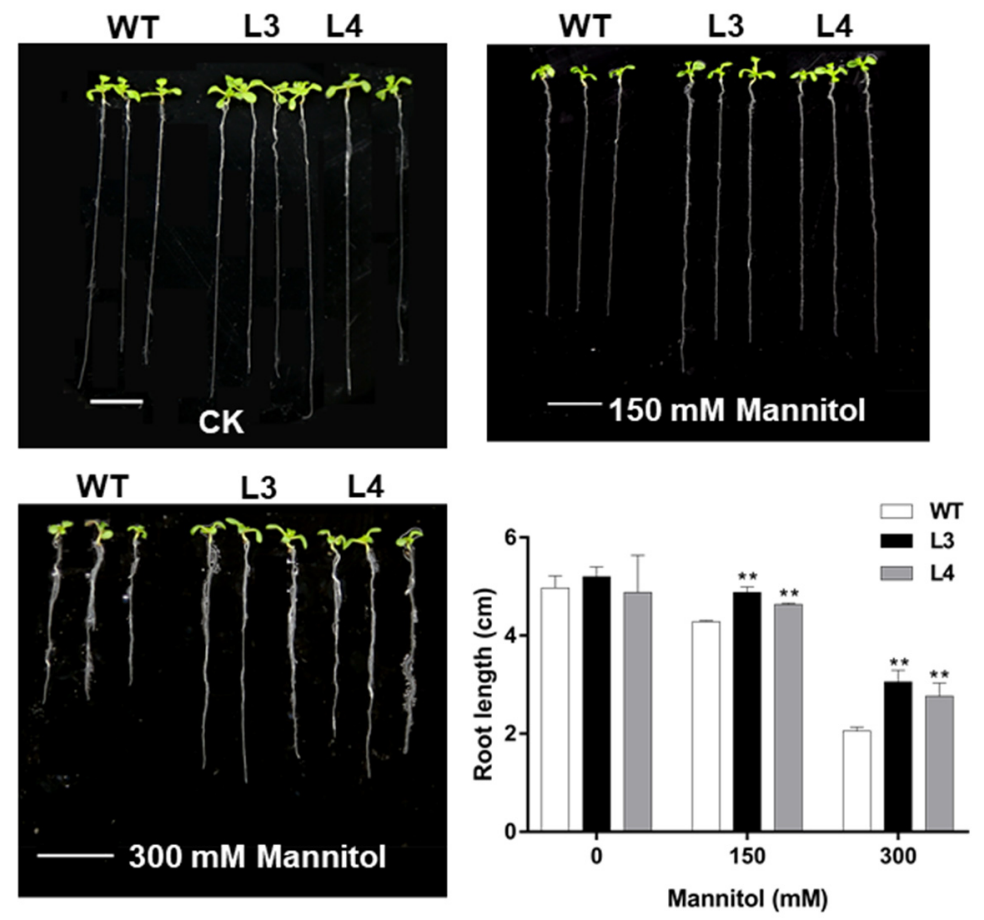

Figure 5. Root elongation analysis of Arabidopsis under mannitol treatment. Error bars represent $\pm \mathrm{SD}$ $(n=3)$. Asterisks denote significant differences (as compared with the control group): ${ }^{* *} p<0.01$.

To study the function of EjWRKY17 in response to drought stress, the five-week-old Arabidopsis seedlings were exposed to drought stress via water withholding. We observed that most plants wilted with symptoms of leaf yellowing and crimping after two weeks of drought treatment (Figure 6A). WT plants exhibited more severe dehydration and wilted phenotype compared to the EjWRKY17 transgenic lines. After rewatering for five days, only $32.5 \%$ of WT plants survived, while over $90 \%$ of the EjWRKY17 transgenic plants returned to normal growth (Figure $6 \mathrm{~B}$ ). Compared to WT plants, a stronger growth was found in the transgenic lines after rewatering. In addition, the water loss rate of detached leaves from WT plants was significantly higher than that of the EjWRKY17 transgenic leaves at five time points over a 10-h time course (Figure 6C). Electrolyte leakage is an important parameter for evaluating cell membrane damage. Under normal conditions, electrolyte leakage was identical between the transgenic lines and WT plants. However, EjWRKY17 overexpression in Arabidopsis exhibited significantly reduced electrolyte leakage than compared to that of the WT after drought treatment (Figure 6D). These results indicated that the overexpression of EjWRKY17 improved the drought tolerance in Arabidopsis plants.

\subsection{Overexpression of EjWRKY17 Decreased ROS and Malondialdehyde (MDA) Accumulation under Drought Stress}

Drought usually causes lipid peroxidation and the excessive production of ROS, ultimately resulting in oxidative stress [29]. In this study, nitroblue tetrazolium (NBT) and $3,3^{\prime}$-diaminobenzidine (DAB) staining were used to visualize the accumulation of $\mathrm{O}^{2-}$ and $\mathrm{H}_{2} \mathrm{O}_{2}$ in Arabidopsis leaves and to investigate whether the drought resistance was associated with the rescue of ROS levels in transgenic lines. As shown in Figure 7A,B, no obvious staining in WT or transgenic plants was observed under control conditions. When exposed to drought stress, lower $\mathrm{O}^{2-}$ and $\mathrm{H}_{2} \mathrm{O}_{2}$ levels were detected in $\mathrm{L} 3$ and $\mathrm{L} 4$ transgenic lines compared to WT plants. In addition, the MDA content in both transgenic and WT plants dramatically increased when drought stress occurred (Figure 7C). According to our data, the transgenic lines consistently accumulated less MDA than WT plants under drought stress, which suggested that overexpression of EjWRKY17 regulated ROS homeostasis and reduced the oxidative damage in Arabidopsis caused by drought stress. 
A

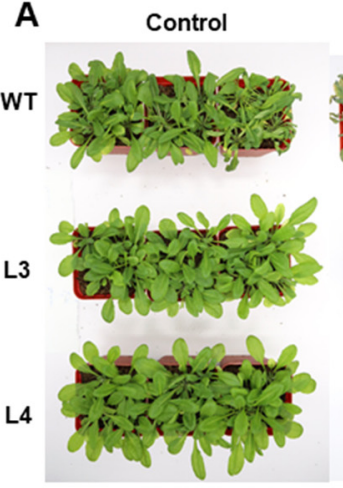

C

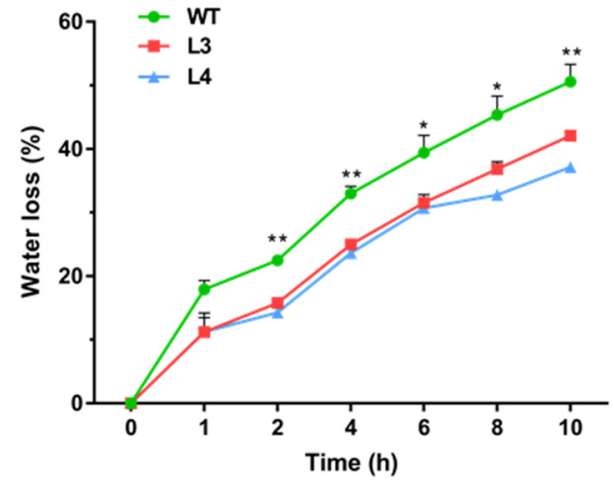

Drought
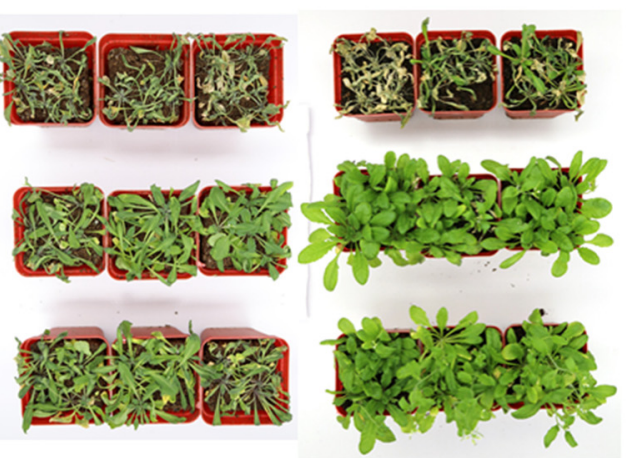

D

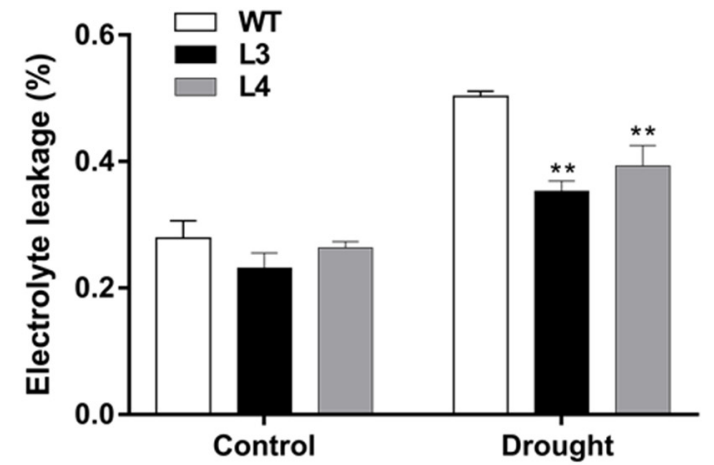

Figure 6. Overexpression of EjWRKY17 confers tolerance to drought stress in Arabidopsis. (A) Phenotype of Arabidopsis with overexpression of EjWRKY17 under water deficit. (B) Survival rates of seedlings. (C) The water loss of detached leaves. (D) Electrolyte leakage. Plants were photographed on $2 \mathrm{~d}$ (control) and $15 \mathrm{~d}$ (drought) without irrigation and on $3 \mathrm{~d}$ after rewatering (rewater). Error bars represent $\pm \mathrm{SD}(n=3)$. Asterisks denote significant differences (as compared with the control group): ${ }^{*} p<0.05 ;{ }^{* *} p<0.01$.
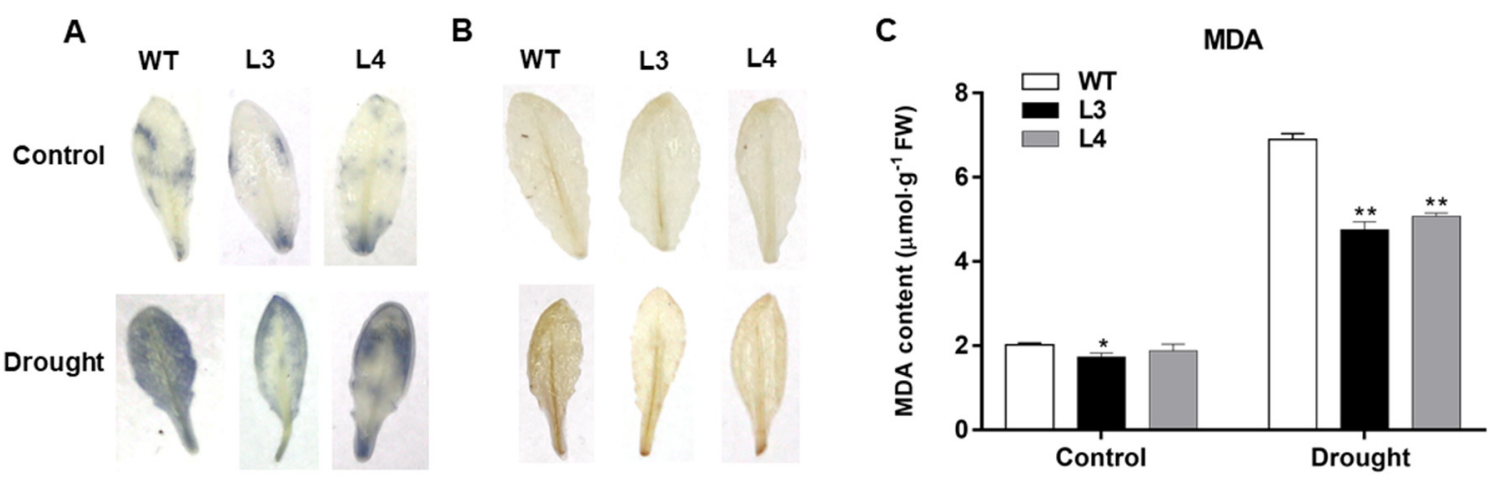

Figure 7. Overexpression of EjWRKY17 decreased ROS production and oxidative damage under drought stress. (A) NBT staining, (B) DAB staining, (C) MDA content. Error bars represent $\pm \mathrm{SD}(n=3)$. Asterisks denote significant differences (as compared with the control group): ${ }^{*} p<0.05 ;{ }^{* *} p<0.01$.

\subsection{Overexpression of EjWRKY17 Positively Regulated ABA-Mediated Stomatal Closure in Arabidopsis}

We further measured stomatal apertures in EjWRKY17 transgenic and WT plants under ABA treatment. Under normal conditions, the stomatal aperture indices of L3 and L4 were significantly higher than those of WT (Figure 8). However, in the presence of $1 \mu \mathrm{M}$ ABA, EjWRKY17 transgenic lines displayed significantly smaller stomatal apertures than 
the control. Thus, our results suggested that overexpression of EjWRKY17 could promote ABA-mediated stomatal closure in Arabidopsis.
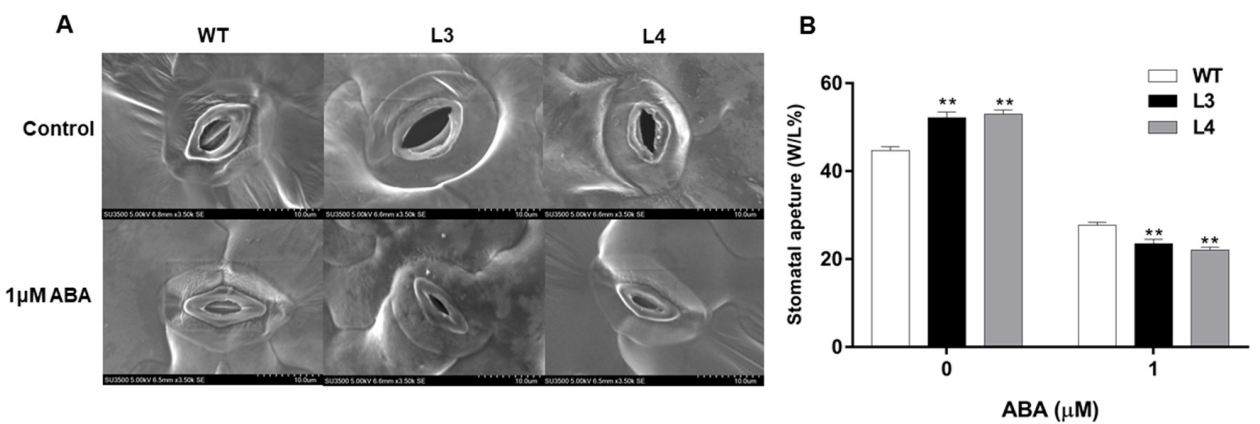

Figure 8. The stomatal aperture of WT and EjWRKY17 transgenic lines under ABA treatment (A,B). Error bars represent $\pm \mathrm{SD}(n=3)$. Asterisks denote significant differences (as compared with the control group): ${ }^{* *} p<0.01$.

\subsection{EjWRKY17 Induced the Expression of Stress-Related Genes under Drought Stress}

To elucidate the effects of EjWRKY17 on the expression of stress-related genes, quantification of the gene expression was conducted. As shown in Figure 9, the expression of AtABF1, AtRD29B, and AtLEA14 genes showed no difference under normal conditions, while transcript levels of these three genes were significantly increased in transgenic lines and WT plants under drought stress. Compared with WT plants, overexpression of EjWRKY17 notably enhanced the transcripts of these genes. In addition, four stressrelated genes (AtRD29A, AtCOR15A, AtRAB18, and AtKIN1) in transgenic lines were prominently up-regulated when seedlings were under normal conditions or exposed to drought conditions. The expression levels of AtRD22 and AtLEA76 in the EjWRKY17overexpressing lines were significantly lower than in WT plants under normal conditions. However, the expression levels of AtRD22 and AtLEA76 were greatly upregulated in the EjWRKY17-overexpressing lines under drought stress, which were significantly higher than in WT plants. To further study how EjWRKY17 regulates these downstream genes, we analyzed the promoter regions of these marker genes with the PlantCARE database (Table S2). One, three, one, three, five, two, and four W-box cis-elements were found in the up-stream sequences of $A B F 1, R D 29 A, R D 29 B, R D 22, L E A 14, L E A 76$, and KIN1, respectively. In addition, two WK box (TTTTCCAC) cis-elements were found in the up-stream sequences of $R A B 18$. Therefore, we speculated that EjWRKY17 may directly or indirectly regulate expression of drought-related genes by binding to the W/WK-box in their promoter regions, thereby responding to drought stress and $\mathrm{ABA}$ signaling. 

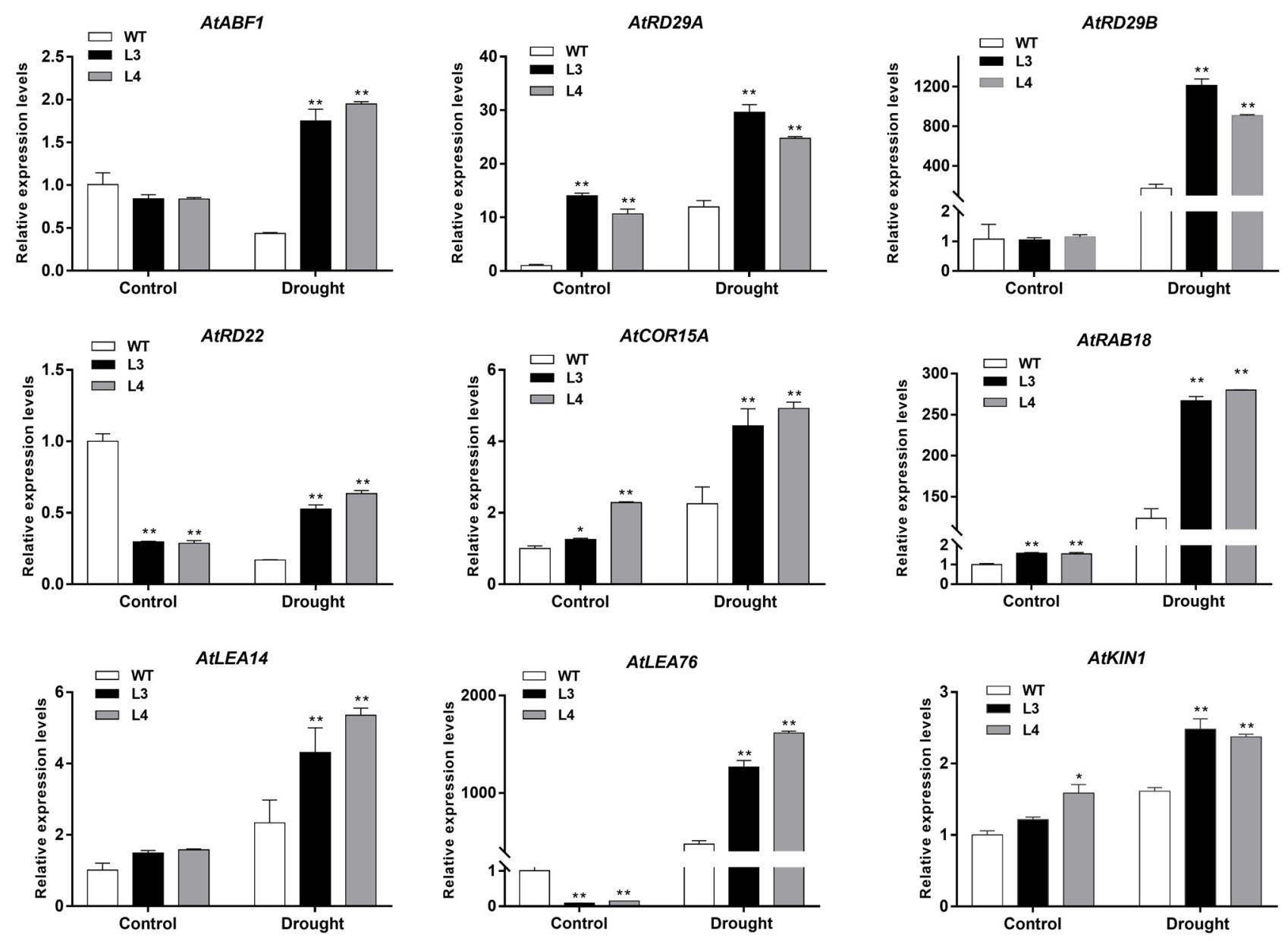

Figure 9. Expression levels of stress-responsive genes in WT and transgenic lines under drought stress. Error bars represent $\pm \mathrm{SD}(n=3)$. Asterisks denote significant differences (as compared with the control group): ${ }^{*} p<0.05 ;{ }^{* *} p<0.01$.

\section{Discussion}

Drought directly affects the growth and productivity of plants. Under drought stress, the total chlorophyll content and photosynthetic rates of loquat leaves were decreased, resulting in a decline in starch content [27]. Severe drought reduced all growth parameters and particularly leaf growth [26]. Our previous research demonstrated that the WRKY family is involved in the enhancement of drought tolerance in loquat seedlings at the transcriptome level. WRKY TFs family are known to mediate abiotic stress responses in various plants; the information of its functions mainly focuses on Arabidopsis [16], rice [20], and soybean [30]. However, the functions and mechanisms of the WRKY gene family in loquat are still unclear. In this study, EjWRKY17, a member of the loquat WRKY family, was characterized to explore its potential role in drought tolerance. The multiple alignments of amino acid sequence and phylogenetic tree analysis of EjWRKY17 exhibited that the EjWRKY17 protein classified into subgroup II shared high similarity with MdWRKY11, MdWRKY17, and other WRKY proteins (PaWRKY11, JrWRKY17, PtWRKY51, and GsWRKY17). Cis-acting elements play an important role in gene transcription and expression [31]. The results showed that many types of cis-acting elements were exhibited in the EjWRKY17 promoter region, including hormone response elements (AAGAA-motif, ABRE, AuxRR-core, CGTCA-motif, ERE, TGACG-motif, CGTCA-motif, MYB-like sequence, and P-box), stress response elements (STRE, TC-rich repeats, 3-AF1, ARE, AE-box, Box 4, LTR, TCCC-motif, WRE3, and as-1), W-box, and plant physiological metabolism-related elements (O2-site, MYB recognition site, circadian, MBS, and GCN4-motif), suggesting that the EjWRKY17 protein was involved in stress and hormone response [32]. The subcellular 
localization assay displayed that the EjWRKY17 protein was localized to the cell nucleus. A previous study reported that WRKY17 was involved in conferring stress tolerance in Arabidopsis [33]. Additionally, The WRKY transcription factors function as positive or negative regulators in salt and drought stress response in different species [23,34]. In the present study, overexpression of EjWRKY17 in Arabidopsis enhanced green cotyledons formation and root elongation under ABA treatment. Besides, the root growth inhibition was alleviated in the transgenic lines under mannitol stresses compared with WT plants. Moreover, the adult transgenic lines showed a distinct resistance to water deficit with a higher survival rate in comparison with WT plants. These results were also confirmed in other plants, such as FtWRKY46 in Tartary buckwheat [6] and HbWRKY82 in Hevea brasiliensis. In addition, overexpression of ZmWRKY17 resulted in decreased ABA sensitivity through regulating the expression of several well-known stress/ABA-responsive genes in Arabidopsis [35]. Our results suggested that ectopic expression of EjWRKY17 resulted in decreased sensitivity to ABA and osmotic stress in Arabidopsis.

The excessive ROS production triggered by drought caused lipid peroxidation, protein oxidation, nucleic acid damage, and even cell death in plants [36]. When loquat seedlings were subjected to drought stress, the MDA content of loquat leaves exhibited an increase followed by a gradual drop [27]. The MDA concentration is a parameter of membrane lipid peroxidation, which reflects the extent of stress [37]. In this study, transgenic plants suffered lower degrees of membrane injury under drought stress in comparison with WT plants, as indicated by reduced accumulation of electrolyte leakage, ROS, and MDA. Hence, these results revealed that the enhanced capacity of the ROS-scavenging system is highly associated with increased drought tolerance in EjWRKY17-overexpressing plants. Our results are consistent with the previous study that IbWRKY2 confers drought resistance in Arabidopsis through activating the ROS-scavenging system [5].

The response pathways to drought mediated by TFs were described as ABA dependent and ABA independent [38]. In the WRKY family, many genes have been reported to participate in the ABA-mediated stress signaling pathway [38]. For instance, WRKY18 and WRKY60 conferred hypersensitivity to ABA stress in inhibiting seed germination and root growth, while WRKY40 antagonized WRKY18 and WRKY60 in response to sensitivity to $\mathrm{ABA}$ and the abiotic stress [39]. The ABA content of loquat leaves gradually accumulated and $A B A$ biosynthetic genes were significantly regulated under drought stress [27]. Moreover, ABA-mediated stomatal closure provided a vital strategy in plant stress responses to prevent water loss [40]. In recent years, several WRKY TFs have been characterized as regulating stomatal closure. The overexpression of GmWRKY16 in Arabidopsis resulted in a water loss rate and reduction of stomatal aperture under drought stress [29]. In a previous study, MfWRKY17 from Myrothamnus flabellifolia was confirmed as a positive regulator to mediate stomatal closure through an ABA-dependent signaling pathway [23]. Similarly, EjWRKY17 overexpression in Arabidopsis led to enhanced tolerance to ABA stress and promoted ABA-induced stomatal closure. Correspondingly, a decrease in the water loss rate was observed in transgenic detached leaves. The results indicate that EjWRKY17 has positive regulatory functions in the drought tolerance of plants through an ABA-dependent signaling pathway.

Extensive research has exhibited that WRKYs TF family positively regulated tolerance to drought stress through up/down-regulating the levels of stress/ABA-responsive genes [13,41]. For instance, overexpression of TaWRKY19 conferred tolerance to salt and drought through up-regulating the expression of $D R E B 2 A, R D 29 A$, and RD29B in transgenic Arabidopsis [42]. RD29A, considered to be a marker gene of the ABA-dependent pathway, is related to reducing stress injury in plants [6]. WRKY57 can directly bind the $\mathrm{W}$-box of RD29A promoter sequences to enhance drought tolerance in Arabidopsis [22]. Overexpression of GmWRKY16 significantly up-regulated the expressions of stress-related marker genes such as RD29A, RD22, KIN1, and LEA14 in transgenic plants under drought stress. Conversely, the negative regulation of WRKY17s in other plant species has been reported. For instance, the expression levels of AtRD29A and AtDREB2B were notably 
down-regulated in CmWRKY17 transgenic Arabidopsis under salinity stress [43]. In contrast, the expression of stress-responsive genes ( $A B F 1, R D 29 A, R D 29 B, R A B 18, R D 22$, and COR15A) and stress-related marker genes (LEA14, LEA76, and KIN1) occurred at much higher levels in these three transgenic lines than in WT plants under drought stresses in this study. In addition, overexpression of HaWRKY76 enhanced drought tolerance of transgenic Arabidopsis lines by regulating the expression of $R A B 18, R D 29 A$, and RD29B [44]. Moreover, GhWRKY59 directly binds to the W-boxes of GhDREB2, which encodes a dehydrationinducible transcription factor regulating ABA-independent drought response [45]. WRKY transcription factors were reported to regulate the expression of target genes by binding to $\mathrm{W}$-box or $\mathrm{WK}$ box in their promoter regions [46]. Hence, we analyzed the promoter sequences of $A B F 1, R D 29 A, R D 29 B, R A B 18, R D 22, L E A 14, L E A 76, K I N 1$, and COR15A with the PlantCARE analysis database, and observed the binding sites existed in the up-stream sequences of these genes except COR15A. We assumed that EjWRKY17 may respond to drought stress by directly or indirectly regulating the drought stress-related genes.

\section{Materials and Methods}

\subsection{Plant Materials}

Grafted Eriobotrya japonica 'Huabai No.1' was potted at the experimental farm of Southwest University, Chongqing, China, and the seedlings were irrigated with water (CK) or melatonin (MT) for $15 \mathrm{~d}$ and then subjected to drought stress by withholding their watering for $13 \mathrm{~d}$, at which point the plants displayed phenotypic foliar wilting [27]. The leaves were collected at 1, 4, 7, 10, and $13 \mathrm{~d}$ and immediately frozen in liquid nitrogen. Nicotiana benthamiana was used for transient expression. Wild type (A. thaliana ecotype) and transgenic plants (homozygous $\mathrm{T} 3$ generation) were cultured in a humidity-controlled environment (16 h light $/ 8 \mathrm{~h}$ dark cycles, $20 \pm 1^{\circ} \mathrm{C}$ ).

\subsection{Cloning and Sequence Analysis of EjWRKY17}

Total RNA extraction, first-strand cDNA synthesis, and qPCR were performed as described previously [47]. The $5^{\prime}$ and $3^{\prime}$ RACE clones were amplified with the SMARTer RACE $5^{\prime} / 3^{\prime}$ Kit (Clontech, Mountain View, CA, USA) and 3'-Full RACE Core Set (TAKARA, Shiga, Japan). The amplicon of partial length was obtained based on the homology cloning, and then the gene was made full length by $5^{\prime}$ and $3^{\prime}$ RACE. The sequences were joined to obtain an open reading frame of EjWRKY17 cDNA. The specific primers are listed in Supplementary Table S3.

The multiple sequence alignment was performed by ClustalX using BioEdit v7.1.11 software (Ibis Biosciences, Carlsbad, CA, USA), and the phylogenetic analysis was constructed with the MEGA software (version 5.0, Auckland, New Zealand) under the Nerghbor-Joining method with 1000 bootstrap replicates [32] with other sequences (Supplementary Table S4) obtained from NCBI (https:/ / www.ncbi.nlm.nih.gov/) (accessed on 30 April 2021). MEME (https:/ / memesuite.org/meme/) (accessed on 30 April 2021) was employed for the analysis of conserved motifs in the WRKY protein. The 2100 bp promoter DNA sequence upstream of the ATG start codon was extracted from the genome (accession No. PRJNA579885) in NCBI. Cis-acting elements in the promoter region were predicted and analyzed using PLANTCARE (http:/ /bioinformatics. psb.ugent.be/webtools/plantcare/html/) (accessed on 10 May 2021).

\subsection{Subcellular Location of EjWRKY17 Protein}

The coding sequence (without stop codon) of EjWRKY17 was cloned into a pCAMBIA 1300-GFP vector and the specific primers with restriction sites (BamHI and XbaI) are listed (Supplementary Table S5). Then, the constructed EjWRKY17-GFP fusion protein and the empty vector were transformed into Agrobacterium tumefaciens strain (GV3101) and transiently expressed in the leaves of Nicotiana benthamiana. The fluorescence signals were captured with a fluorescence microscope Observer DP80 (Olympus, Tokyo, Japan). 


\subsection{Arabidopsis Transformation}

The coding sequence of EjWRKY17 was cloned into the overexpression vector pFGC5941 with CaMV $35 S$ promoters to generate the recombinant vector. Then, the resultant construct was introduced into Agrobacterium tumefaciens strain (GV3101) and transformed into Arabidopsis Col-0 by the floral dip method [48]. The transgenic lines were screened by $20 \mathrm{mg} / \mathrm{L}$ glufosinateammoniums and confirmed by PCR analysis (Table S5 and Figure S2). Two T3 homozygous lines (L3 and L4) with relatively higher expression were selected for observing phenotype and further analysis.

\subsection{Seedling Growth Assays}

For seed germination assays, seeds of homozygous T3 transgenic lines and WT were sown on half-strength MS agar media with ABA $(0,0.5$, and $1.0 \mu \mathrm{M})$. Seeds were stratified at $4{ }^{\circ} \mathrm{C}$ for 2 days under darkness and then moved to a climate-controlled room at $16-\mathrm{h}$ light. 8-h dark cycle and $23{ }^{\circ} \mathrm{C}$ for four days.

For root elongation assays, Arabidopsis seedlings were grown normally on half-strength MS media for four days. Seedlings with similar growth status were then transferred to half-strength media containing ABA $(0,1.0$, and $5.0 \mu \mathrm{M})$ and mannitol $(0,150$, and $300 \mathrm{mM})$, respectively. The length of the primary root was recorded after 4 days with three replications.

\subsection{Drought Stress Tolerance Assays}

For drought tolerance assays, four-week-old soil-grown transgenic EjWRKY17 lines and WT plants under normal conditions were stopped irrigating until stress symptoms occurred, and the seedlings were then re-watered for recovery. For the determination of electrolyte leakage, sample leaves $(0.1 \mathrm{~g})$ were rinsed in $10 \mathrm{~mL}$ of distilled water, and then the electrolyte leakage in the solution (E1) was measured after $22 \mathrm{~h}$ of floating at room temperature by conductivity meter (DDS- $309^{+}$, Chengdu, China). Subsequently, the leaves were boiled for $20 \mathrm{~min}$. After the solution had cooled to room temperature, the conductivity of the solution (E2) was recorded. The relative electrolyte leakage was evaluated as (E1/E2) $\times 100 \%$ [49]. Water loss data were recorded for ten detached leaves of WT and transgenic Arabidopsis lines at $0,0.5,1,2,4,6,8$, and $12 \mathrm{~h}$.

\subsection{Measurement of the Content of ROS and MDA}

$\mathrm{DAB}$ and NBT staining were performed to detect the accumulation of $\mathrm{H}_{2} \mathrm{O}_{2}$ and $\mathrm{O}^{2-}$ as previously described [50]. Meanwhile, the MDA content was determined according to the previous study [51].

\subsection{Stomatal Aperture Analysis}

For stomatal aperture assay, rosette leaves of four-week-old Arabidopsis seedlings were collected and incubated in $200 \mathrm{~mL}$ stomatal closure solution $(10 \mathrm{mM} \mathrm{KCl}, 1 \mathrm{mM}$ $\mathrm{CaCl}_{2}, 25 \mathrm{mM}$ MES) for $2 \mathrm{~h}$ [52]. Photographs were taken using an optical microscope (Hitachi, Tokyo, Japan). The width to length ratio of stomatal was calculated using ImageJ software (version 1.8.0, National Institutes of Health; Bethesda, MD, USA).

\subsection{Quantitative Real-Time Polymerase Chain Reaction ( $q R T-P C R$ )}

qRT-PCR was conducted to measure changes in stress-related gene expression by using qTOWER ${ }^{3} \mathrm{G}$ (Analytik Jena, Jena, Germany). PCR amplification procedures was set as following: $95{ }^{\circ} \mathrm{C}$ for $30 \mathrm{~s}$, followed by 40 cycles at $95{ }^{\circ} \mathrm{C}$ for $20 \mathrm{~s}, 56{ }^{\circ} \mathrm{C}$ for $60 \mathrm{~s}$, and a melt cycle from 65 to $95^{\circ} \mathrm{C}$. All primer sequences are listed in Supplementary Table S6 and the loquat qEjActin gene and Arabidopsis AtActin gene were used as internal controls. PCRs were performed in $10-\mu \mathrm{L}$ volumes using NovoStart ${ }^{\circledR}$ SYBR qPCR SuperMix Plus (Novoprotein, Shanghai, China) with each primer at a concentration of $0.05 \mu \mathrm{M}$. The $2^{-\Delta \Delta \mathrm{Ct}}$ method [53] was used to calculated the relative expression values. 


\subsection{Statistical Analysis}

Each experiment was based on three independent biological replicates of each sample and three technical replicates of each biological replicate. The data were shown as the means \pm SD. Significant analysis was evaluated by Student's t-test and SPSS 19.0 software (SPSS Inc., Chicago, IL, USA) with One-way ANOVAs analysis.

\section{Conclusions}

In summary, our results suggest that EjWRKY17 is a drought-related TF located in the nucleus. Overexpression of EjWRKY17 decreased the ABA sensitivity level of transgenic Arabidopsis. Moreover, the inhibition of water loss rate, electrolyte leakage, ROS, and MDA accumulation further confirmed the enhanced drought tolerance in the EjWRKY17 transgenic lines. Furthermore, we also proved that EjWRKY17 improved tolerance to drought in transgenic Arabidopsis by promoting ABA-induced stomatal closure and activating stress-related genes. Our data provide insights into the regulation of drought tolerance by EjWRKY17, while the function of EjWRKY17 in response to other stresses needs to be further investigated.

Supplementary Materials: The following are available online at https:/ / www.mdpi.com/article/10 .3390/ijms22115593/s1.

Author Contributions: Conceptualization, D.W., D.J. and G.L.; methodology, D.W. and X.L.; formal analysis, W.C.; investigation, D.W. and Q.C.; resources, G.L. and Q.G.; data curation, D.W. and Q.C.; writing—original draft preparation, D.W.; writing—-review and editing, W.C. and D.J.; supervision, G.L.; project administration, Y.X.; funding acquisition, D.J., Q.G. and G.L. All authors have read and agreed to the published version of the manuscript.

Funding: This research was funded by National Key Research and Development Program of China (No. 2019YFD1000200), Fundamental Research Funds for the Central Universities (XDJK2019AA001, XDJK2020B058 and XDJK2019D040), and the Innovation Research Group Funds for Chongqing Universities (CXQT19005).

Institutional Review Board Statement: Not applicable.

Informed Consent Statement: Not applicable.

Data Availability Statement: EjWRKY17 sequence data is available in GenBank.

Conflicts of Interest: The authors declare no conflict of interest.

\section{References}

1. Gao, T.; Zhang, Z.; Liu, X.; Wu, Q.; Chen, Q.; Liu, Q.; van Nocker, S.; Ma, F.; Li, C. Physiological and transcriptome analyses of the effects of exogenous dopamine on drought tolerance in apple. Plant Physiol. Biochem. 2020, 148, 260-272. [CrossRef]

2. Zhang, Y.; Zhou, Y.; Zhang, D.; Tang, X.; Li, Z.; Shen, C.; Han, X.; Deng, W.; Yin, W.; Xia, X. PtrWRKY75 overexpression reduces stomatal aperture and improves drought tolerance by salicylic acid-induced reactive oxygen species accumulation in poplar. Environ. Exp. Bot. 2020, 176, 104117. [CrossRef]

3. Ju, Y.L.; Min, Z.; Yue, X.F.; Zhang, Y.L.; Zhang, J.X.; Zhang, Z.Q.; Fang, Y.L. Overexpression of grapevine VvNAC08 enhances drought tolerance in transgenic Arabidopsis. Plant Physiol. Biochem. 2020, 151, 214-222. [CrossRef] [PubMed]

4. Yu, Y.; Bai, Y.; Wang, P.; Wang, Y.; Wan, H.; Liu, C.; Ni, Z. Soybean nuclear factor YA10 positively regulates drought resistance in transgenic Arabidopsis thaliana. Environ. Exp. Bot. 2020, 180, 104249. [CrossRef]

5. Zhu, H.; Zhou, Y.; Zhai, H.; He, S.; Zhao, N.; Liu, Q. A novel sweetpotato WRKY transcription factor, IbWRKY2, positively regulates drought and salt tolerance in transgenic Arabidopsis. Biomolecules 2020, 10, 506. [CrossRef] [PubMed]

6. Lv, B.; Wu, Q.; Wang, A.; Li, Q.; Dong, Q.; Yang, J.; Zhao, H.; Wang, X.; Chen, H.; Li, C. A WRKY transcription factor, FtWRKY46, from Tartary buckwheat improves salt tolerance in transgenic Arabidopsis thaliana. Plant Physiol. Biochem. 2020, 147, 43-53. [CrossRef]

7. Mei, F.; Chen, B.; Li, F.; Zhang, Y.; Kang, Z.; Wang, X.; Mao, H. Overexpression of the wheat NAC transcription factor TaSNAC4-3A gene confers drought tolerance in transgenic Arabidopsis. Plant Physiol. Biochem. 2021, 160, 37-50. [CrossRef]

8. Meng, C.; Sui, N. Overexpression of maize MYB-IF35 increases chilling tolerance in Arabidopsis. Plant Physiol. Biochem. 2019, 135, 167-173. [CrossRef]

9. Yao, P.; Sun, Z.; Li, C.; Zhao, X.; Li, M.; Deng, R.; Huang, Y.; Zhao, H.; Chen, H.; Wu, Q. Overexpression of Fagopyrum tataricum FtbHLH2 enhances tolerance to cold stress in transgenic Arabidopsis. Plant Physiol. Biochem. 2018, 125, 85-94. [CrossRef] 
10. Rushton, D.L.; Tripathi, P.; Rabara, R.C.; Lin, J.; Ringler, P.; Boken, A.K.; Langum, T.J.; Smidt, L.; Boomsma, D.D.; Emme, N.J.; et al. WRKY transcription factors: Key components in abscisic acid signalling. Plant Biotechnol. J. 2012, 10, 2-11. [CrossRef]

11. Bao, W.; Wang, X.; Chen, M.; Chai, T.; Wang, H. A WRKY transcription factor, PcWRKY33, from Polygonum cuspidatum reduces salt tolerance in transgenic Arabidopsis thaliana. Plant Cell Rep. 2018, 37, 1033-1048. [CrossRef] [PubMed]

12. Wang, Y.; Jiang, L.; Chen, J.; Tao, L.; An, Y.; Cai, H.; Guo, C. Overexpression of the alfalfa WRKY11 gene enhances salt tolerance in soybean. PLoS ONE 2018, 13, e0192382. [CrossRef]

13. Li, W.; Pang, S.; Lu, Z.; Jin, B. Function and mechanism of WRKY transcription factors in abiotic stress responses of plants. Plants 2020, 9, 1515. [CrossRef] [PubMed]

14. Dong, Q.; Zheng, W.; Duan, D.; Huang, D.; Wang, Q.; Liu, C.; Li, C.; Gong, X.; Li, C.; Mao, K.; et al. MdWRKY30, a group IIa WRKY gene from apple, confers tolerance to salinity and osmotic stresses in transgenic apple callus and Arabidopsis seedlings. Plant Sci. 2020, 299, 110611. [CrossRef]

15. Zhang, Y.; Yu, H.; Yang, X.; Li, Q.; Ling, J.; Wang, H.; Gu, X.; Huang, S.; Jiang, W. CsWRKY46, a WRKY transcription factor from cucumber, confers cold resistance in transgenic-plant by regulating a set of cold-stress responsive genes in an ABA-dependent manner. Plant Physiol. Biochem. 2016, 108, 478-487. [CrossRef] [PubMed]

16. Zhao, K.X.; Chu, S.S.; Zhang, X.D.; Wang, L.P.; Rono, J.K.; Yang, Z.M. AtWRKY21 negatively regulates tolerance to osmotic stress in Arabidopsis. Environ. Exp. Bot. 2020, 169, 103920. [CrossRef]

17. Li, J.; Besseau, S.; Toronen, P.; Sipari, N.; Kollist, H.; Holm, L.; Palva, E.T. Defense-related transcription factors WRKY70 and WRKY54 modulate osmotic stress tolerance by regulating stomatal aperture in Arabidopsis. New Phytol. 2013, 200, 457-472. [CrossRef] [PubMed]

18. Ding, Z.J.; Yan, J.Y.; Xu, X.Y.; Yu, D.Q.; Li, G.X.; Zhang, S.Q.; Zheng, S.J. Transcription factor WRKY46 regulates osmotic stress responses and stomatal movement independently in Arabidopsis. Plant J. 2014, 79, 13-27. [CrossRef] [PubMed]

19. Gao, H.; Wang, Y.; Xu, P.; Zhang, Z. Overexpression of a WRKY transcription factor TaWRKY2 enhances drought stress tolerance in transgenic wheat. Front. Plant Sci. 2018, 9, 997. [CrossRef]

20. Lee, H.; Cha, J.; Choi, C.; Choi, N.; Ji, H.S.; Park, S.R.; Lee, S.; Hwang, D.J. Rice WRKY11 plays a role in pathogen defense and drought tolerance. Rice 2018, 11, 5. [CrossRef] [PubMed]

21. Arnao, M.B.; Hernandez-Ruiz, J. Melatonin in flowering, fruit set and fruit ripening. Plant Reprod. 2020, 33, 77-87. [CrossRef] [PubMed]

22. Jiang, Y.; Liang, G.; Yu, D. Activated expression of WRKY57 confers drought tolerance in Arabidopsis. Mol. Plant 2012, 5, 1375-1388 [CrossRef]

23. Huang, Z.; Guo, H.D.; Liu, L.; Jin, S.H.; Zhu, P.L.; Zhang, Y.P.; Jiang, C.Z. Heterologous expression of dehydration-inducible MfWRKY17 of Myrothamnus flabellifolia confers drought and salt tolerance in Arabidopsis. Int. J. Mol. Sci. 2020, 21, 4603. [CrossRef]

24. Zhang, L.; Yu, H.; Lin, S.Q.; Gao, Y.S. Molecular characterization of FT and FD homologs from Eriobotrya deflexa Nakai forma koshunensis. Front. Plant Sci. 2016, 7, 8. [CrossRef] [PubMed]

25. Cao, S.; Yang, Z.; Zheng, Y. Sugar metabolism in relation to chilling tolerance of loquat fruit. Food Chem. 2013, 136, 139-143. [CrossRef] [PubMed]

26. Gugliuzza, G.; Talluto, G.; Martinelli, F.; Farina, V.; Lo Bianco, R. Water deficit affects the growth and leaf metabolite composition of young loquat plants. Plants 2020, 9, 274. [CrossRef] [PubMed]

27. Wang, D.; Chen, Q.; Chen, W.; Guo, Q.; Xia, Y.; Wang, S.; Jing, D.; Liang, G. Physiological and transcription analyses reveal the regulatory mechanism of melatonin in inducing drought resistance in loquat (Eriobotrya japonica Lindl.) seedlings. Environ. Exp. Bot. 2021, 181, 104291. [CrossRef]

28. Xu, J.; Trainotti, L.; Li, M.; Varotto, C. Overexpression of isoprene synthase affects ABA- and drought-related gene expression and enhances tolerance to abiotic stress. Int. J. Mol. Sci. 2020, 21, 4276. [CrossRef] [PubMed]

29. Wei, Z.; Gao, T.; Liang, B.; Zhao, Q.; Ma, F.; Li, C. Effects of exogenous melatonin on methyl viologen-mediated oxidative stress in apple leaf. Int. J. Mol. Sci. 2018, 19, 316. [CrossRef]

30. Zhou, Q.Y.; Tian, A.G.; Zou, H.F.; Xie, Z.M.; Lei, G.; Huang, J.; Wang, C.M.; Wang, H.W.; Zhang, J.S.; Chen, S.Y. Soybean WRKY-type transcription factor genes, GmWRKY13, GmWRKY21, and GmWRKY54, confer differential tolerance to abiotic stresses in transgenic Arabidopsis plants. Plant Biotechnol. J. 2008, 6, 486-503. [CrossRef]

31. Meng, D.; Li, Y.; Bai, Y.; Li, M.; Cheng, L. Genome-wide identification and characterization of WRKY transcriptional factor family in apple and analysis of their responses to waterlogging and drought stress. Plant Physiol. Biochem. 2016, 103, 71-83. [CrossRef]

32. Karkute, S.G.; Gujjar, R.S.; Rai, A.; Akhtar, M.; Singh, M.; Singh, B. Genome wide expression analysis of WRKY genes in tomato (Solanum lycopersicum) under drought stress. Plant Gene 2018, 13, 8-17. [CrossRef]

33. Ali, M.A.; Azeem, F.; Nawaz, M.A.; Acet, T.; Abbas, A.; Imran, Q.M.; Shah, K.H.; Rehman, H.M.; Chung, G.; Yang, S.H.; et al. Transcription factors WRKY11 and WRKY17 are involved in abiotic stress responses in Arabidopsis. J. Plant Physiol. 2018, 226, 12-21. [CrossRef]

34. Yan, H.; Jia, H.; Chen, X.; Hao, L.; An, H.; Guo, X. The cotton WRKY transcription factor GhWRKY17 functions in drought and salt stress in transgenic Nicotiana benthamiana through ABA signaling and the modulation of reactive oxygen species production. Plant Cell Physiol. 2014, 55, 2060-2076. [CrossRef] [PubMed] 
35. Cai, R.; Dai, W.; Zhang, C.; Wang, Y.; Wu, M.; Zhao, Y.; Ma, Q.; Xiang, Y.; Cheng, B. The maize WRKY transcription factor ZmWRKY17 negatively regulates salt stress tolerance in transgenic Arabidopsis plants. Planta 2017, 246, 1215-1231. [CrossRef] [PubMed]

36. Li, X.; Tang, Y.; Li, H.; Luo, W.; Zhou, C.; Zhang, L.; Lv, J. A wheat R2R3 MYB gene TaMpc1-D4 negatively regulates drought tolerance in transgenic Arabidopsis and wheat. Plant Sci. 2020, 299, 110613. [CrossRef]

37. Li, T.; Huang, Y.; Khadr, A.; Wang, Y.H.; Xu, Z.S.; Xiong, A.S. DcDREB1A, a DREB-binding transcription factor from Daucus carota, enhances drought tolerance in transgenic Arabidopsis thaliana and modulates lignin levels by regulating lignin-biosynthesis-related genes. Environ. Exp. Bot. 2020, 169, 103896. [CrossRef]

38. Banerjee, A.; Roychoudhury, A. WRKY proteins: Signaling and regulation of expression during abiotic stress responses. Sci. World J. 2015, 2015, 807560. [CrossRef]

39. Bidabadi, S.S.; VanderWeide, J.; Sabbatini, P. Exogenous melatonin improves glutathione content, redox state and increases essential oil production in two Salvia species under drought stress. Sci. Rep. 2020, 10, 6883. [CrossRef]

40. Qu, L.; Sun, M.; Li, X.; He, R.; Zhong, M.; Luo, D.; Liu, X.; Zhao, X. The Arabidopsis F-box protein FOF2 regulates ABA-mediated seed germination and drought tolerance. Plant Sci. 2020, 301, 110643. [CrossRef]

41. Ren, X.; Chen, Z.; Liu, Y.; Zhang, H.; Zhang, M.; Liu, Q.; Hong, X.; Zhu, J.K.; Gong, Z. ABO3, a WRKY transcription factor, mediates plant responses to abscisic acid and drought tolerance in Arabidopsis. Plant J. 2010, 63, 417-429. [CrossRef] [PubMed]

42. Niu, C.F.; Wei, W.; Zhou, Q.Y.; Tian, A.G.; Hao, Y.J.; Zhang, W.K.; Ma, B.; Lin, Q.; Zhang, Z.B.; Zhang, J.S.; et al. Wheat WRKY genes TaWRKY2 and TaWRKY19 regulate abiotic stress tolerance in transgenic Arabidopsis plants. Plant Cell Environ. 2012, 35, 1156-1170. [CrossRef] [PubMed]

43. Li, P.; Song, A.; Gao, C.; Wang, L.; Wang, Y.; Sun, J.; Jiang, J.; Chen, F.; Chen, S. Chrysanthemum WRKY gene CmWRKY17 negatively regulates salt stress tolerance in transgenic chrysanthemum and Arabidopsis plants. Plant Cell Rep. 2015, 34, 1365-1378. [CrossRef] [PubMed]

44. Raineri, J.; Ribichich, K.F.; Chan, R.L. The sunflower transcription factor HaWRKY76 confers drought and flood tolerance to Arabidopsis thaliana plants without yield penalty. Plant Cell Rep. 2015, 34, 2065-2080. [CrossRef]

45. Li, F.; Li, M.; Wang, P.; Cox, K.L., Jr.; Duan, L.; Dever, J.K.; Shan, L.; Li, Z.; He, P. Regulation of cotton (Gossypium hirsutum) drought responses by mitogen-activated protein (MAP) kinase cascade-mediated phosphorylation of GhWRKY59. New Phytol. 2017, 215, 1462-1475. [CrossRef]

46. Rushton, P.J.; Somssich, I.E.; Ringler, P.; Shen, Q.J. WRKY transcription factors. Trends Plant. Sci. 2010, 15, 247-258. [CrossRef]

47. Chen, W.; Wang, P.; Wang, D.; Shi, M.; Xia, Y.; He, Q.; Dang, J.; Guo, Q.; Jing, D.; Liang, G. EjFRI, FRIGIDA (FRI) Ortholog from Eriobotrya japonica, delays flowering in Arabidopsis. Int. J. Mol. Sci. 2020, 21, 1087. [CrossRef]

48. Clough, S.J.; Bent, A.F. Floral dip: A simplified method for Agrobacterium-mediated transformation of Arabidopsis thaliana. Plant J. 1998, 16, 735-743. [CrossRef]

49. Quan, W.; Hu, Y.; Mu, Z.; Shi, H.; Chan, Z. Overexpression of AtPYL5 under the control of guard cell specific promoter improves drought stress tolerance in Arabidopsis. Plant Physiol. Biochem. 2018, 129, 150-157. [CrossRef]

50. Yang, X.; He, K.; Chi, X.; Chai, G.; Wang, Y.; Jia, C.; Zhang, H.; Zhou, G.; Hu, R. Miscanthus NAC transcription factor MlNAC12 positively mediates abiotic stress tolerance in transgenic Arabidopsis. Plant Sci. 2018, 277, 229-241. [CrossRef]

51. Kumar, C.N.M.; Knowles, N.R. Changes in lipid peroxidation and lipolytic and free-radical scavenging enzyme activities during aging and sprouting of potato (Solanum tuberosum) seed-tubers. Plant Physiol. 1993, 102, 115-124. [CrossRef] [PubMed]

52. Zhang, Y.; Wan, S.; Liu, X.; He, J.; Cheng, L.; Duan, M.; Liu, H.; Wang, W.; Yu, Y. Overexpression of CsSnRK2.5 increases tolerance to drought stress in transgenic Arabidopsis. Plant Physiol. Biochem. 2020, 150, 162-170. [CrossRef] [PubMed]

53. Livak, K.J.; Schmittgen, T.D. Analysis of relative gene expression data using real-time quantitative PCR and the $2^{-\Delta \Delta C T}$ method. Methods 2001, 25, 402-408. [CrossRef] [PubMed] 